\title{
Diagnóstico e Continuidade do Cuidado do Câncer Bucal em pacientes acompanhados pelas Equipes de Saúde Bucal do Programa de Saúde da Família: a experiência do município de Resende, no Estado do Rio de Janeiro
}

\author{
Continuity of Care Diagnostics and Oral Cancer in patients followedby \\ oral health teams from the Family Health Program: theexperience of the \\ municipality of Resende, State of Rio de Janeiro
}

Artigo

Original

Original

Paper

Sylvio da Costa Júnior ${ }^{1}$

Carlos Gonçalves Serra ${ }^{2}$

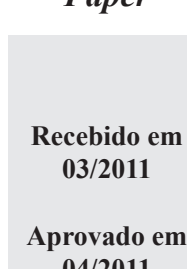

\section{Palavras-chave: \\ Câncer de boca \\ Continuidade do \\ Cuidado}

Programa de Saúde da Família

\section{Resumo}

Foi realizado um estudo qualitativo em Equipes de Saúde Bucal (ESB) do Programa de Saúde da Família (PSF), no Município de Resende, no Estado do Rio de Janeiro, visando avaliar a integralidade da assistência e a continuidade do cuidado à saúde para o câncer bucal. Em função da importância do câncer bucal e das condições das ESB do PSF em poder fazer o diagnóstico precoce, este trabalho se propõe a estudar, no município, as condições de diagnóstico e continuidade do cuidado para o câncer bucal. O objetivo deste estudo é analisar as condições técnicas e operacionais dos cirurgiões dentistas das ESB para identificação, diagnóstico precoce e encaminhamento de pacientes cadastrados nas suas áreas de atuação, com suspeita de lesões orais consideradas pré-cancerosas. Para obtenção dos dados primários, foram utilizadas técnicas de entrevistas, com roteiros semiestruturados Assim, quantitativamente tivemos: 11 dentistas do PSF no município de Resende e os profissionais do CEO. A entrevista semiestruturada, parte do trabalho de campo, foi elaborada contendo perguntas fechadas e abertas. Após levantamento e consolidação dos dados qualitativos e quantitativos, podemos constatar que o município conta com uma rede de saúde articulada, em que os pacientes com casos de lesões suspeitas são encaminhados para o centro de referência para continuidade do cuidado. Entretanto, podemos observar que a cobertura de $45,94 \%$ da população de Resende pelas Equipes de Saúde Bucal, correspondendo a 59.722 habitantes, se constitui num fator limitante para a detecção precoce de lesões suspeitas de câncer bucal. Dessa forma, torna-se importante a ampliação da oferta de serviços de saúde bucal, objetivando não só aumentar a resolubilidade, evitando transtornos adicionais aos pacientes, como dar mais agilidade ao diagnóstico, contribuindo para a qualidade na sobrevida dos pacientes com diagnóstico positivo de câncer oral. 
It was performed a qualitative study on oral health teams (ESB) of the Family Health Program (FHP) in the municipality of Resende, State of Rio de Janeiro, to evaluate the integrality care and continuity of health care for mouth cancer. Because of the importance of oral cancer and conditions of the PSF in ESB it was performed early diagnosis, this study aims to examine the conditions of continuity of care and diagnosis for oral cancer in the county. The aim of this study is to analyze the technical and operational conditions of the dental surgeons of ESB for identification, early diagnosis and referral of patients enrolled in their fields, with suspected oral lesions are considered pre-cancerous. To obtain the primary data were used interviewing techniques, using semi-structured Thus, we had quantitatively: 11 dentists in the city of Resende PSF and the professionals of CEO. The semi-structured interview, part of the fieldwork, was prepared with closed and open questions. After gathering and consolidating qualitative and quantitative data it was possible to find that the city has a articulated healthcare network, where cases of patients with suspicious lesions are sent to the reference center for continuity of care. In spite of the low coverage of ESB it was possible to find out that the expansion of health services by Primary Care, and consequently, the increase of the resolvability, avoiding additional disorders for patients and giving faster diagnosis, can, effectively contribute to the quality on survival of patients with positive diagnosis of oral cancer.

Mouth Cancer

Continuity of Care

Family Health

Program

\section{Introdução}

A denominação câncer de boca inclui os cânceres de lábio e de cavidade oral. A incidência do câncer bucal no Brasil é uma das mais altas do mundo e os baixos índices de sobrevida refletem o seu comportamento agressivo, exigindo, desta forma, por parte dos profissionais, maior atenção a esta neoplasia, tanto em relação à prevenção quanto ao diagnóstico e acompanhamento. A maioria dos estudos envolvendo lesões orais não separa as regiões específicas dentro da cavidade oral, no entanto, o câncer de lábio é mais frequente em pessoas brancas e registra maior ocorrência no lábio inferior em relação ao superior. $O$ câncer em outras regiões da boca acomete principalmente os tabagistas, sendo que os riscos aumentam quando estes também são etilistas (BUNDGAARD, BENTZEN \& WILDT, 1994; COTRAN, KUMAR, COLLINS, 2000).

Aproximadamente $10 \%$ dos tumores malignos que ocorrem no corpo humano estão localizados na boca (PINHEIRO, 1998), sendo esse o sexto tipo de câncer mais incidente no mundo (SYRJANEN, 2005). Excluindose o câncer de pele, o câncer bucal pode ser considerado o mais comum da região de cabeça e pescoço, com incidência de 38\%
(CARVALHO et al., 2001; DEDIVITIS et al., 2004), com predominância no sexo masculino, e $75 \%$ dos casos diagnosticados na faixa etária dos 60 anos (COSTA et al., 2002).

Segundo Cotran, Kumar, Collins (2000) e Há \& Caetano (2004), cerca de 95\% dos casos são carcinomas epidermoides. O câncer de boca é o oitavo tipo de câncer mais frequente entre os homens (9.985 casos estimados/ano) e o nono entre as mulheres (3.895 casos estimados/ano) e suas taxas de incidência e mortalidade vêm aumentando, sendo consideradas das mais altas do mundo (BRASIL, 2004).

A questão da prevenção do câncer bucal é bastante complexa em virtude da multiplicidade de fatores envolvidos, inclusive culturais, tornando difícil o controle. História familiar, fatores sócio-demográficos, tipo de dieta e estilo de vida, todos são coparticipantes, porém, a capacidade de intervenção nesses fatores é limitada (DEBS YD, 2000). Em contrapartida, a forma mais eficaz e simples de combate a esse câncer, particularmente, é pela ênfase na promoção de saúde, aumento do acesso aos serviços de saúde e diagnóstico precoce.

Em função da importância do câncer bucal a das condições das Equipes de Saúde Bucal (ESB) do Programa Saúde da Família (PSF) em poder fazer o diagnóstico precoce, 
este trabalho se propõe a estudar as condições de diagnóstico e continuidade do cuidado para o câncer bucal, no município de Resende, no Estado do Rio de Janeiro.

\section{Fundamentação Teórica}

\subsection{A Construção do Modelo Odontológico de Atenção em Saúde}

Em 1923, o Presidente Artur Bernardes promulgou a Lei ${ }^{\circ} 4.682$, de 24 de Janeiro, de autoria do Deputado Eloy Chaves, que institui o Seguro Social no Brasil (MERCADANTE, 2002). Com o advento dessa lei, a partir de 1923, surgiram as Caixas de Aposentadoria e Pensões - CAPs. Essas Caixas eram organizadas por categorias de classe e empresas, públicas ou privadas, que ofertavam serviços pecuniários e de saúde aos seus contribuintes, inclusive medicamentos. Os recursos das Caixas de Aposentadoria e Pensões eram pagos por contribuições obrigatórias de empregados e empregadores. A gerência desses recursos e a administração dos serviços prestados eram organizadas por uma comissão com representantes de patrões e empregados. Ao Governo cabia apenas dirimir conflitos (MERCADANTE, 2002).

Com o crescimento das CAPs e com o volume de recursos crescente que cada CAPs administra, em 1930 surgem os IAPs Institutos de Aposentadorias e Pensões. A diferença marcante entre os CAPs e os IAPs é o aumento significativo da participação estatal.

Na saúde pública, a odontologia começou a crescer a partir de 1933 com a criação dos IAPs. Originalmente, os serviços tinham um caráter meramente mutilador, visando somente a remoção de focos infecciosos e não havia o cuidado com a adoção de ferramentas que visassem a promoção de saúde. Por insuficiência do conhecimento da época, não se evitou os complexos desdobramentos decorrentes das mutilações que interferem muito nos intrincados jogos da socialização, para além dos aspectos epidemiológicos associados (ZANETTI, 1993). A odontologia, inserida nos IAPs, assim como os demais ramos da medicina estavam orientados a atender os setores produtivos da emergente classe industrializa- da nacional, padrão nada preocupado com o chamado bem estar social.

Grandes diferenças de gestão e financeiras entre os IAPs motivaram movimentos da época contra e a favor para a unificação dos Institutos. Na década de 60, o debate sobre a unificação ou não dos IAPs foi intenso: de um lado trabalhadores a favor da unificação com o argumento de tornar os Institutos menos desiguais, e por outro lado, trabalhadores contra a unificação, sob o argumento de que a unificação representava a perda de participação dos trabalhadores e o risco de maior centralização do poder estatal (MERCADENTE, 2002).

Segundo Roncalli (1999), na medida em que os IAPs cresciam e financeiramente se encorpavam, aumentava a centralização e influência estatal e diminuía a participação dos trabalhadores. Assim, o Estado brasileiro passou a ser o maior financiador desse modelo de proteção social com o setor privado sendo o principal prestador de serviço.

De acordo com Groismam, Moraes e Chagas (2005), o Estado adotou um modelo de atenção à saúde que privilegiava o setor privado em detrimento de investimentos na rede a atenção à saúde pública, modelo este chamado por muitos de privatista, em que a rede privada de saúde se robusteceu e se agigantou, a partir de recursos públicos, através, principalmente, de convênios. Esse modelo continuou a privilegiar somente os trabalhadores formais com "carteira assinada".

Em 1966, quando os Institutos foram unificados, ao longo de mais de 30 anos de um modelo de Odontologia Previdenciária, foram definidos protocolos uniformes de atendimento às populações previdenciárias. Entretanto, as ferramentas institucionais utilizadas e o Ato Normativo $n^{\circ} 47$ de 17 de outubro de 1969 continuaram nivelando por baixo o atendimento odontológico, dando ênfase ao modelo restaurador-mutilador de atendimento, via pagamento somente a atendimentos clínicos invasivos. A novidade do Ato Normativo foi a inclusão da odontopediatria entre as possibilidades de atendimento de livre demanda, incluindo como procedimento a profilaxia à cárie dentária.

A expansão orçamentária da Previdência na década de 70 possibilitou o crescimento do atendimento odontológico, com aumento 
significativo da oferta de serviços, ainda que dentro do modelo de atendimento clínico da época. As políticas de saúde bucal tinham um forte foco na ampliação do acesso à prática profissional, ocorrendo, em consequência, um maciço credenciamento de dentistas que trabalhavam em clínicas privadas. Não era objeto de preocupação dos gestores de saúde bucal conceitos e modelos baseados na equidade e universalidade de atendimento, ou da qualidade do serviço prestado, a ênfase era na ampliação da oferta, eminentemente privada (ZANETTI, 1993).

Esse serviço era ofertado aos trabalhadores urbanos pelo INPS, aos trabalhadores rurais pelo FUNRURAL, àqueles que estavam fora do mercado de trabalho, eventualmente por religiosos e, aos bancários, pelo Serviço de Assistência e Seguro Social dos Economiários (PINTO, 1977). Nesse período, a administração Federal continuou preterindo o Ministério da Saúde (MS) como coordenador nacional das políticas de proteção sanitário-bucais. Ainda no início da crise financeira que se abateu sobre a Previdência Social em 1977- segundo estimativa- $5 \%$ dos gastos da União com saúde eram destinados à odontologia (US\$ 120 milhões). Destes, o percentual de menos de 1\% se encontrava no MS. Em 1981, mais de $58 \%$ dos gastos públicos estavam na esfera do INAMPS, os estados tinham $40,1 \%$ e o MS $1,1 \%$. Nesse ano, segundo estimativas, a iniciativa privada correspondia a $81,8 \%$ do total de recursos movimentados pela odontologia (VIANNA, 1988).

O chamado "Movimento Sanitário Odontológico", no bojo das políticas reformistas de saúde de então, lutou tanto pela transformação do modelo hegemônico de atenção à saúde bucal quanto pela redemocratização da sociedade brasileira. O movimento reformista da saúde, no qual o movimento odontológico e suas conferências se inserem é paralelo e complementar ao movimento de redemocratização brasileiro (SERRA, 1998).

A odontologia previdenciária sofreu- assim como a medicina previdenciária- as mudanças e dificuldades provenientes do projeto de saúde pública privatista da década de 70 (SERRA, 1998), do aumento da cobertura do setor de seguro saúde do setor privado, da rápida modernização e incorporação de apare- lhos e insumos odontológicos e da mercantilização de todo setor dentário (SERRA, 1998; ZANETTI, 1993).

\subsection{A Estratégia Saúde da Família: princípios básicos}

Na década de 1990, foram editadas pelo Ministério da Saúde as Normas Operacionais Básicas visando à definição de objetivos e estratégias para a orientação da dinâmica operacional do SUS, isto é: a consolidação da descentralização do SUS, sua municipalização e reorientação do modelo de atenção. Assim, as Normas Operacionais Básicas, NOB/SUS91,93 e 96, objetivavam redefinir:

1. Os papéis de cada esfera de governo;

2. O caráter da direção única;

3. Ferramentas para que Estados e Municípios superem o mero papel de prestadores de serviços de saúde e passem assumir o papel de gestores;

4. O papel de fluxos de financiamento, reduzindo o pagamento por procedimentos e direcionando a transferência de recursos para transferências fundo a fundo.

5. O papel da participação e controle social sobre o sistema de saúde.

Dos preceitos advindos do Programa Saúde da Família, a territorialização das unidades de saúde com suas respectivas responsabilidades sanitárias representa um importante instrumento para a continuidade do cuidado.

Dentro dessa lógica, a integração e a continuidade do cuidado consistem em uma estruturação permanente das práticas clínicas voltadas para o usuário do sistema, visando garantir a globalidade dos serviços requeridos de diferentes profissionais nos variados níveis do sistema.

\subsection{O Papel dos Cirurgiões-Dentistas da Atenção Primária e do PSF}

Leavell e Clark, em sua obra "Medicina Preventiva", publicado em 1976, nortearam conceitualmente a chamada medicina preventiva. A prevenção é colocada em três estágios (primária, secundária e terciária) e cada estágio ligado a um período clínico (pré-patogê- 
nese, patogênese e sequela) e a um nível de atuação. A prevenção primária deve acontecer no período de pré-patogênese, onde existem as condições para o estabelecimento de patologias ligadas ao fator de risco associado. Dentro desse conceito, cada nível de prevenção está ligado a um nível de aplicação ou atuação.

A Saúde Bucal, cada dia mais, desponta como preocupação, tanto no enfoque da promoção e prevenção, quanto assistencial. Os agravos bucais e suas sequelas são de grande prevalência no Brasil, constituindo-se em graves problemas de saúde pública, com consequências sociais e econômicas.

O tratamento oncológico, de modo geral, necessita de recursos médicos-hospitalares mais complexos e sofisticados, envolvendo, muitas das vezes mutilações com complicações estéticas e de movimentação de músculos da cabeça e do pescoço.

Dessa maneira, estudos que investiguem a associação entre indicadores de atenção primária à saúde bucal e condições de acesso e resolubilidade são particularmente importantes para a formulação de políticas de saúde direcionadas a reduzir desigualdades em saúde, sobretudo na utilização de serviços.

$\mathrm{O}$ atraso relativo à precocidade do diagnóstico refere-se também ao encaminhamento, que podemos considerar como o tempo entre a chegada do paciente ao clínico da atenção primária até o momento do possível diagnóstico final de câncer bucal. Estabelece-se, dessa forma, uma relação direta com o sistema que organiza os fluxos de encaminhamento para a realização do diagnóstico (ONIZAWA et al. 2003; PITIPHAT et al., 2002).

As ações de saúde em odontologia vêm crescendo muito Brasil desde a sua inclusão no Programa Saúde da Família, realizada no ano de 2000, pela portaria/MS no 1444 do Ministério da Saúde.

O CEO, que é atenção especializada em odontologia de nível secundário, oferece para a população serviços de diagnóstico bucal, com ênfase no diagnóstico e detecção do câncer de boca, periodontia especializada, cirurgia oral menor dos tecidos moles e duros, endodontia e atendimento a portadores de necessidades especiais (LETÁCIO, 2009).

\subsection{O "Brasil Sorridente"}

Orientado pela política de regionalização, o Ministério da Saúde criou, em 2003, o Programa Brasil Sorridente, com o claro objetivo de garantir a integralidade do atendimento e a continuidade do cuidado bucal através do CEO, nível secundário de atenção odontológica, que oferece exames e consultas especializadas em Saúde Bucal.

Dessa maneira, a porta de entrada desse usuário do sistema de saúde público municipal deverá ser o Programa de Saúde da Família com Equipe de Saúde Bucal, para, nos casos de maior complexidade, haver o encaminhamento para os Centros de Especialidades Odontológicas (CEOs), garantido, assim, a integralidade das ações.

O objetivo é que esses Centros de Especialidades Odontológicas possam, no nível médio de complexidade, dar suporte para a atenção primária, quer nas unidades tradicionais quer nas Equipes de Saúde Bucal do Programa Saúde da Família, sendo unidades de referencias para a atenção primária, trabalhando de acordo com a realidade epidemiológica do território sanitário.

Os Centros de Especialidades Odontológicas-incluídosnoCadastroNacional de Estabelecimentos de Saúde (CNES), e classificados como Clínicas Especializadas e Ambulatórios de Especialidade -estão estruturados para ofertar à população, no mínimo, as seguintes especialidades:

- diagnóstico bucal, com ênfase no diagnóstico e detecção do câncer oral;

- $\quad$ periodontia especializada;

- cirurgia oral menor;

- endodontia e

- atendimento a pacientes portadores de necessidades especiais.

\subsection{O Câncer de Boca}

De todas as lesões cancerígenas na cavidade oral o carcinoma epidermoide (ou carcinoma espinocelular ou carcinoma de células escamosas) corresponde a $90 \%$ a $94 \%$ do total. Mostra-se como uma úlcera assintomática- que aumenta de tamanho e não cicatrizaque pode apresentar bordas elevadas, firmes e

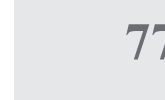

\section{.}

(1)

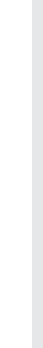

.

\section{(1)}
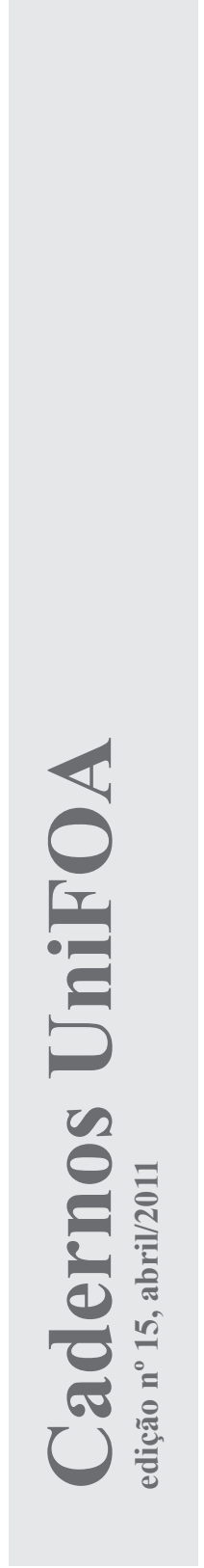
endurecidas. Seu diagnóstico deve ser baseado em achados histológicos e pode ser classificado como muito, moderadamente ou pouco diferenciado.

\section{Problema, Objetivos e Método}

\subsection{Problema}

Em função da alta incidência do câncer bucal no Brasil e dos baixos índices de sobrevida, o que demonstra a agressividade desta patologia, há necessidade, cada vez maior, de que o profissional das equipes de saúde bucal no PSF esteja capacitado para diagnosticar precocemente lesões suspeitas de câncer oral e dar continuidade ao cuidado.

\subsection{Objetivos}

\subsubsection{Objetivo Geral}

Analisar as condições técnicas e operacionais dos cirurgiões dentistas das Equipes de Saúde Bucal do PSF do Município de Resende, no Estado do Rio de Janeiro, para identificação, diagnóstico precoce e encaminhamento de pacientes cadastrados nas suas áreas de atuação, com suspeita de lesões orais consideradas pré-cancerosas.

\subsubsection{Objetivos Específicos}

- Conhecer e descrever a estrutura dos serviços de apoio diagnóstico e tratamento do câncer bucal dos usuários encaminhados pelas Equipes de Saúde Bucal do Programa Saúde da Família do município de Resende.

- Identificar e analisar as dificuldades das unidades de referência para diagnóstico e tratamento do câncer bucal no município de Resende.

\subsection{Métodos e procedimentos}

\subsubsection{Coleta de dados}

\section{- Primários}

Para obtenção dos dados primários foram utilizadas técnicas de entrevistas, com roteiros semiestruturados. Assim, quantitativamente tivemos onze dentistas do PSF no município de Resende. A amostra incluiu dois odontólogos, especialistas do Centro de Especialidades Odontológicas de Resende, para enriquecer a análise sobre a continuidade do cuidado. Dessa forma, esses dois profissionais do Centro de Referência foram incluídos na amostra, porém, na análise dos resultados, estes odontólogos do CEO ficaram à parte. A entrevista semiestruturada, parte do trabalho de campo, foi elaborada contendo perguntas fechadas e abertas.

Cada questionário aplicado aos participantes do estudo continha explicações sobre a natureza do estudo e o Termo de Consentimento Livre e Esclarecido a ser por eles preenchido, caso concordassem em participar da pesquisa.

Os dados secundários foram obtidos através das referências bibliográficas e documentais, que possibilitaram embasamento às questões teóricas do trabalho.

\subsubsection{Cenário do estudo}

\section{Município de Resende}

O município de Resende conta com aproximadamente 130.000 habitantes (IBGE, 2007); 01 Hospital Municipal de Emergência com 47 leitos; 01 Hospital Geral Filantrópico/ Santa Casa com 70 leitos; 01 Maternidade Filantrópica/APMIR com 78 leitos, totalizando 195 leitos públicos; uma rede de saúde de 22 Módulos de Saúde da Família implan$\operatorname{tados}^{1}, 17$ Equipes de Saúde Bucal, todas na modalidade I, 01 Centro de Especialidades Odontológicas tipo II (Portaria n²17/GM, de 11 de fevereiro de 2005) para onde são encaminhados os casos de atenção secundária odontológica. 
Segundo a portaria $648 \mathrm{GM} / \mathrm{MS}$, de 28 de Março de 2006:

São itens necessários à implantação das Equipes de Saúde da Família:

I - existência de equipe multiprofissional responsável por, no máximo, 4.000 habitantes, sendo a média recomendada de 3.000 habitantes, com jornada de trabalho de 40 horas semanais para todos os seus integrantes (...).

Com isso, a cobertura de Equipes de Saúde Bucal no município é de 45,94\% da população. Segundo cadastro no Ministério da Saúde, há no município 119 Agentes Comunitários de Saúde cadastrados, cobrindo 63.825 habitantes, o que corresponde a $49,08 \%$ de cobertura ${ }^{2}$. O sistema municipal de saúde de Resende conta com outros níveis de complexidade, tendo ainda um hospital Geral, especializado em atendimento de urgência com 9 leitos de CTI; 01 hemonúcleo com capacidade diária de atendimento de 40 pessoas/dia; um centro municipal de reabilitação e Fisioterapia, com média anual de atendimento de 23.800 pessoas, sendo a média de atendimento hospitalar anual de 68.500 pessoas.

Como determina o artigo $9^{\circ}$ da Lei 8080 de setembro de 1990, a Secretaria Municipal de Saúde de Resende é a gestora dos serviços de saúde ofertados a população.

(...) a direção do Sistema Único de Saúde (SUS) é única, de acordo com o inciso I do art. 198 da Constituição Federal, sendo exercida em cada esfera de governo pelos seguintes órgãos: I - no âmbito da União, pelo Ministério da Saúde; II - no âmbito dos Estados e do Distrito Federal, pela respectiva Secretaria de Saúde ou órgão equivalente; e III - no âmbito dos Municípios, pela respectiva Secretaria de Saúde ou órgão equivalente.

E segundo o artigo ${ }^{\circ} 18$ da mesma Lei:

À direção municipal do Sistema de Saúde (SUS) compete: I - planejar, organizar, controlar e avaliar as ações e os serviços de saúde e gerir e executar os serviços públicos de saúde; II - participar do planejamento, programação e organização da rede regionalizada e hierarquizada do Sistema Único de Saúde (SUS), em articulação com sua direção estadual (...);

\subsection{Aspectos Éticos}

Inicialmente, este estudo foi submetido à análise pelo Comitê de Ética em Pesquisa da Universidade Estácio de Sá (UNESA), seguindo os requisitos preconizados pela Resolução 196/96 do Conselho Nacional de Saúde (CNS). Solicitou-se também anuência da Secretaria Municipal de Saúde de Resende para a efetivação deste estudo. A participação dos sujeitos da pesquisa, dentistas da rede municipal que trabalham na ESF, se deu de forma voluntária, mediante solicitação de permissão para a gravação e a explicitação sobre a finalidade e importância da colaboração, seguida da assinatura no Termo de Consentimento Livre e Esclarecido.

\section{Resultados e Discussão}

Deve-se assinalar que as unidades da Fazenda da Barra II, Fazenda da Barra III e Itapuca estavam em obras; 01 dentista (CD) estava em férias, 01profissional trabalhava na zona rural em mais de um Módulo de PSF e não foi contactada e 01 profissional de ESB não se sentiu à vontade para falar sobre o tema.

Os profissionais que aceitaram participar deste estudo assinaram o Termo de Consentimento Livre e Esclarecido, autorizando que suas respostas fossem analisadas. Os dentistas entrevistados representam 64,70\% do total de dentistas pertencentes às ESB. Como três unidades estavam em reforma, podemos dizer que o universo de entrevistados representa 78,57\% do total de dentistas em atividade em ESF. Alonge e Narendran (2003) em estudo similar com dentistas obtiveram percentual de $40 \%$ de participação do públicoalvo. Na pesquisa destes autores o questionário foi encaminhado via correio para os dentistas, na expectativa de devolução do mesmo pelos

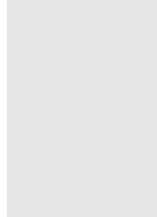

(⿸丆口

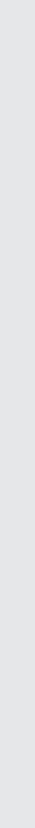


participantes, diferente deste presente estudo em que o pesquisador foi a campo realizar as entrevistas.

\subsection{Tema: Perfil dos entrevistados}

\subsubsection{Categoria: Participação}

No tocante ao perfil profissional, o estudo reuniu informações de 11 dentistas do PSF (representam 64,70 \% dos profissionais de ESB do município) referentes aos conhecimentos sobre o tema. Para enriquecer a análise sobre a integralidade da assistência e a continuidade do cuidado, foram entrevistados 2 $(50 \%)$ odontológos especialista em cirurgia buço-macilo-facial do CEO, no universo de 4 especialistas em cirurgia, que são os responsáveis no Centro de Referência pela biópsia em lesões suspeitas de câncer oral. Convém ressaltar que a análise quantitativa dos dados será realizada somente com os odontógos do PSF.

A participação dos dentistas do CEO se deu em caráter complementar para enriquecimento da análise sobre a continuidade do cuidado. Alonge e Narendran (2003) em pesquisa realizada com dentistas sobre câncer bucal obtiveram $40 \%$ de participação dos odontólogos contactados, levando-os a conclusão que havia pouco interesse dos dentistas sobre o tema.

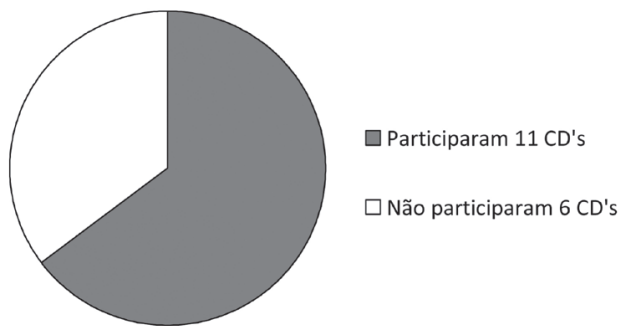

Gráfico 1. Distribuição dos cirurgiões-dentistas (CDs) do PSF segundo a participação no estudo.

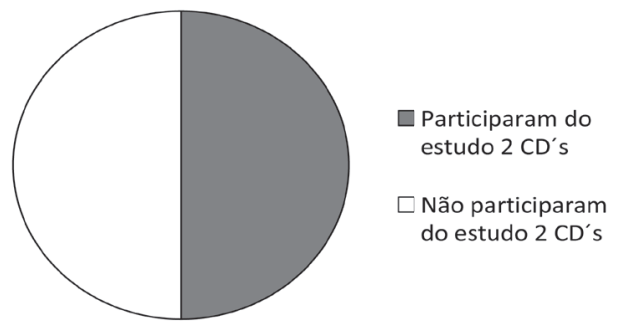

Gráfico 2. Distribuição dos cirurgiões-dentistas (CDs) do CEO segundo a participação no estudo. 4.1.2 Categoria: Idade

Em relação à idade dos dentistas (gráfico 3) entrevistados $04(36,4 \%)$ têm entre $20 \mathrm{e}$
30 anos; $05(45,4 \%)$ entre 30 e 40 anos e 02 $(18,2 \%)$ com 40 anos ou mais.

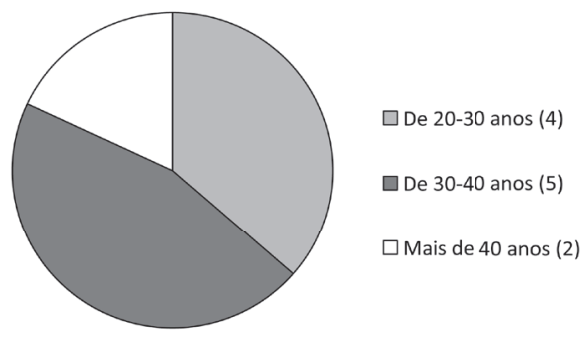

Gráfico 3. Idade dos CDs entrevistados

\subsubsection{Categoria: tempo de graduado}

Conforme sintetiza o gráfico 04, a pesquisa identificou, em relação ao tempo de graduado dos entrevistados, que 02 dentistas $(18,2 \%)$ têm 01 ano ou menos de graduado; 02 $(18,2 \%)$ entre 01 e 05 anos; $04(36,4 \%)$ com mais de 05 e menos de 10 anos de graduado e 03 dentistas $(27,3 \%)$ com mais de 10 anos de graduado. Os resultados desta pesquisa em relação aos anos de graduação diferem de outros trabalhos, como o realizado por Alonge e Narendran (2003) e Kujan et al. (2006) na qual os odontólogos com mais de 20 anos de graduação eram percentualmente maiores quando comparados com outras faixas de idades, nos respectivos estudos.

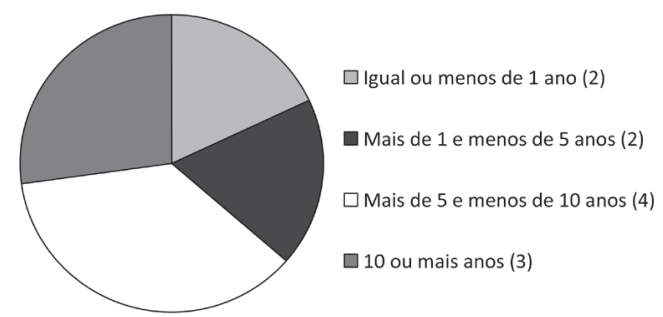

Gráfico 4. Tempo de graduado dos CDs entrevistados (em anos)

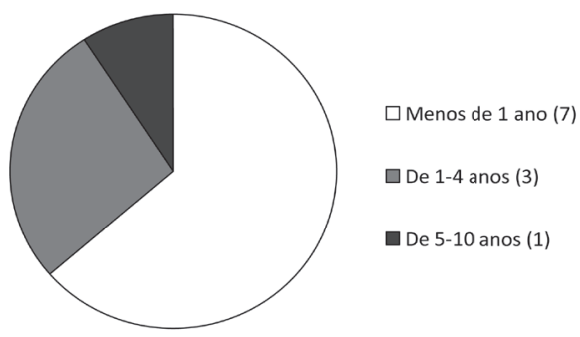

Grafico 5. Tempo de trabalho em PSF (em anos)

Assim, dos dentistas entrevistados, o maior percentual $(36,36 \%)$ refere-se aos profissionais (quatro) com mais de 5 anos e menos de 10 anos de graduação e, quanto à idade, $45,45 \%$ (cinco) estão na faixa entre $30 \mathrm{e}$ 40 anos. McCann et al. (2000) no seu estudo 
com dentistas sobre câncer de boca na Escócia encontrou um percentual de $33 \%$ de dentistas com mais de 20 anos de graduado.

\subsubsection{Categoria: especialização}

No que diz respeito aos dentistas possuirem ou não especializações (gráfico 06), $05(45,45 \%)$ responderam que possuem alguma especialização, enquanto $06(54,54 \%)$ não possuem qualquer especialização.

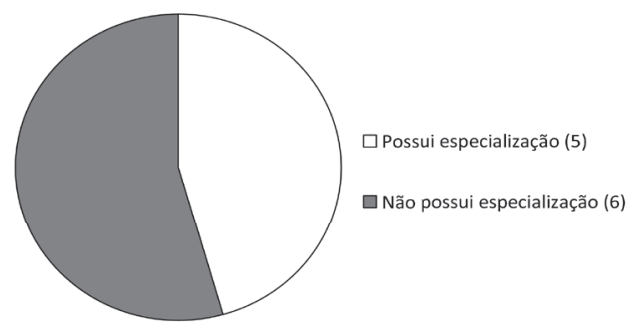

Grafico 6. Distribuição dos CDs com e sem especialização.

Como 54,54\% dos entrevistados não possuem especialização, estes profissionais podem ser caracterizados como generalistas. Kujan et al. (2006) em estudo com dentistas sobre câncer oral identificou majoritariamente como generalistas mais de $70 \%$ dos dentistas entrevistados.

\subsubsection{Categoria: vínculo com o PSF}

A pesquisa identificou que 06 dentistas $(54,55 \%)$ trabalham exclusivamente no Módulo de Saúde da Família no município de Resende, não possuindo qualquer outro tipo vínculo, enquanto 05 dentistas $(45,45 \%)$ relataram ter outro tipo de trabalho, além daquele em que desempenham suas funções no Programa de Saúde da Família. Dos CDs que relataram outro tipo de trabalho, todos, ou seja, 100\% disseram trabalhar em consultorios particulares com outros colegas de profissão. Kujan et al. (2006) encontrou um percentual de $20 \%$ de dentistas que trabalham somente na serviço público, quando o questionamento foi sobre se havia outros vínculos de trabalho.

Como a Portaria/MS nº48, de 28 de Março de 2006, que regulamenta o Programa de Saúde da Família preconiza dedicação de 40 horas semanais de trabalho, constatamos que $54,55 \%$ dos dentistas trabalham exclusivamente no PSF.
$\mathrm{Na}$ pesquisa desenvolvida por Leão et al. (2005) o número de dentista com outro vínculo de trabalho foi de $62 \%$, além do compromisso na atenção primária.

Um dos dentistas entrevistados, que possui vínculo empregatício classificado como estatutário, justificou sua dedicação exclusiva ao PSF da seguinte maneira: “(...) a Prefeitura paga um bom salário, que me permite ficar aqui 8 horas por dia".

Em relação ao vínculo empregatício, dos 11 profissionais do PSF, 01é contratado via Cruz Vermelha, 03 são cargos de confiança e 07 foram admitidos por concurso público, realizado em 2010. Dessa forma, somente os admitidos por concurso público e, assim, submetidos ao Estatuto dos Servidores Municipais, fazem jus à gratificação por trabalharem no Programa de Saúde da Família e, por isto, têm salário diferenciado em comparação aos outros profissionais que também trabalham no PSF.

Cruzando estes dados podemos perceber que dos 07 dentistas admitidos em concurso público, 02 (28,57\%) tem outro vínculo de trabalho, além do PSF, e $05(71,42 \%)$ trabalham exclusivamente no PSF. A relação se inverte quando analisamos os dentistas classificados como não estatutários, em que dos 04 dentistas não estatutários, apenas 01 (25\%) dedica todo seu tempo de trabalho ao Programa de Saúde da Família.

Um dos dentistas não estatutários que possui outro vínculo trabalhista, arguido sobre o porquê de não trabalhar exclusivamente no PSF respondeu que “(...) o salário não é tão bom para viver só dele e eu posso ser mandado embora a qualquer momento".

Dessa maneira, o perfil dos profissionais sujeitos deste estudo, pode ser registrado da seguinte forma: possuem entre 30 a 40 anos $(45,45 \%)$ de idade; de 05 a 10 anos de graduado $(36,36 \%) ; 81,8 \%$ s são do sexo feminino; $54,54 \%$ são generalistas; $72,72 \%$ possuem vínculo trabalhista, classificados como servidor público do Município e 06 (54,54\%) dentistas dedicam todo seu tempo e trabalho profissional exclusivamente ao Programa de Saúde da Família.

Quanto a questão de gênero por parte dos pesquisados, este estudo coincide com pesquisa realizada por Leão et al. (2005), na qual houve predomínio de dentistas mulheres 
(52\%), porém com percentual muito inferior ao apresentado neste estudo $(81,81 \%)$.

\subsection{Tema - diagnóstico precoce do cân- cer bucal pelas ESB/PSF}

\subsubsection{Categoria: capacitação institucional}

Em relação à esta categoria, $10(90,9 \%)$ dentistas relataram que não tiveram nenhum tipo de capacitação institucional sobre diagnóstico precoce de câncer de boca e apenas 01 $(9,1 \%)$ confirmou ter tido capacitação institucional sobre o tema. Entretanto, 09 (81,8\%) afirmaram que, ao longo de sua tragetória profissional, fizeram algum tipo de capacitação sobre o tema, enquanto $02(18,2 \%)$ dos entrevistados disseram que nunca participaram de cursos específicos sobre o tema.

Algumas falas dos dentistas entrevistados retratam, de forma bem semelhante, a situaçào da maioria $(81,8 \%)$ em relação à capacitação sobre câncer bucal. Assim, como exemplo: “(...) eu sempre participo de Congressos ou cursos gratuitos realizados pela $\mathrm{ABO}$ e na faculdade sempre tinha Jornadas Universitárias".

Todos os dentistas (100\%) entrevistados disseram que a Prefeitura sempre realiza cursos de capacitação com os dentistas da rede municipal, embora nenhum abordasse tema "câncer de boca". Os dentistas relataram que " a Prefeitura e a Secretaria de Saúde sempre realizam cursos, constantemente, mas ainda não teve um sobre câncer de boca, mas sempre tem curso para fazer pela Prefeitura".

\subsubsection{Subcategoria: Participação em atividades acadêmicas sobre câncer oral}

Quanto à participação dos dentistas em atividades acadêmicas sobre $\mathrm{o}$ tema relacionado com câncer bucal, verificou-se que 2 disseram que haviam participado de cursos sobre $o$ tema a menos de 1 ano; 6 dentistass relataram ter participados de cursos sobre o tema a mais de 1 anos e 3 dentistas nunca participaram de cursos sobre o tema. Para a maioria dos entrevistados $(90,9 \%)$ a atualização sobre câncer bucal ocorreu em Congressos Científicos, onde participaram por conta própria.
Os 11 dentistas entrevistados (100\%) relataram que gostariam de participar de cursos de capacitação sobre o diagnóstico precoce de câncer de boca. Esta fala é representativa dessa posição geral: " gostaria de participar de capacitação sobre câncer de boca sim, pois me ajudaria muito na clínica e para eu poder continuar me atualizando".

\subsubsection{Subcategoria: Condições técni- cas e operacionais}

Sobre este aspecto, $10(90,9 \%)$ dentistas relataram ter condições técnicas e conhecimento profissional para deteç̧ão de lesões suspeitas de malignidade oral. $\mathrm{O}$ mesmo percentual de dentistas $(90,9 \%)$ dos Módulos de ESB relataram que as unidades lhes oferecem boas condições físicas, de material e de insumos para um bom atendimento clínico e para uma boa investigação para rastreamento de lesões pré-malignas ou malignas. Apenas 01 $(9,1 \%)$ dentista relatou que sua unidade poderia ter um ar condicionado, mais espaço e, consequentemente, conforto para o profissional e paciente: “(...) no verão, o consultório odontológico é muito quente, o que afeta a qualidade do trabalho".

Quanto à experiência de estar diante de uma possível lesão sugestiva de malignidade de câncer oral, 04 (36,36\%) dentistas disseram que já se depararam com este tipo de lesão oral e a conduta de todos foi a de encaminhar os pacientes para o Centro de Referência de Especialidades Odontológicas do município (CEO) para que, após avaliação do especialista, estes pacientes ficassem à disposição para uma possível biópsia incisional ou exciosional e exames complementares. Esta fala reflete a conduta dos profissionais : “(...) eu mediquei o paciente com analgésicos e anti-inflamatório para aliviar a dor local e encaminhei para o Centro de Especialidades".

Todos $(100 \%)$ os dentistas pesquisados relataram que não faltam insumos para o atendimento aos pacientes “(...) aqui não falta luva, material de consumo, nem nada que possa paralisar o atendimento. Sempre que pedimos material a entrega é muito rápida". 


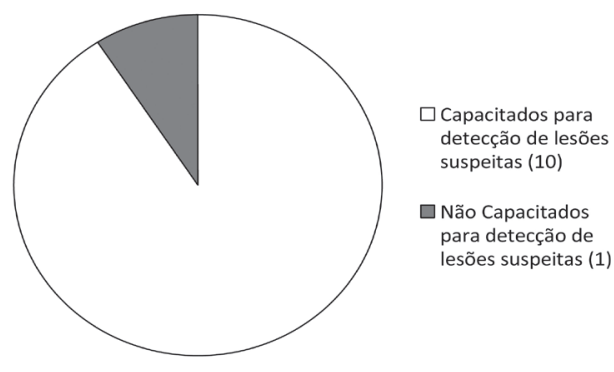

Gráfico 7. Percepção dos CDs segundo a capacitação para deteç̧ão de lesões suspeitas de malignidade oral.

\subsubsection{Subcategoria: fatores de risco}

Quando apresentados ao quadro contendo vários fatores de risco e arguidos sobre o grau de importância de cada um deles no que diz respeito à etiologia do câncer bucal e o grau de importância de cada condição oral em relação à evolução do câncer bucal, as respostas foram distribuídas por número de citações, conforme sintetizado no quadro abaixo:

\begin{tabular}{|l|c|c|c|c|}
\hline & Importante & $\begin{array}{c}\text { Moderadamente } \\
\text { Importante }\end{array}$ & Sem Importância & Não sabe \\
\hline Idade do Paciente & 7 & 4 & 0 & 0 \\
\hline Álcool & 11 & 0 & 0 & 0 \\
\hline Infecção bacteriana & 4 & 4 & 1 & 2 \\
\hline Trauma & 7 & 3 & 2 & 2 \\
\hline Infecção Fúngica & 2 & 5 & 0 & 0 \\
\hline Tabagismo & 11 & 0 & 1 & 2 \\
\hline Infecção Viral & 3 & 5 & 1 & 0 \\
\hline
\end{tabular}

\begin{tabular}{|l|c|c|c|c|}
\hline & Importante & $\begin{array}{c}\text { Moderadamente } \\
\text { Importante }\end{array}$ & Sem Importância & Não Sabe \\
\hline Aftas & 3 & 7 & 1 & 0 \\
\hline Candidíase & 4 & 4 & 3 & 0 \\
\hline Eritroplasia & 7 & 4 & 0 & 0 \\
\hline Tatuagem por Mercúrio & 1 & 3 & 1 & 0 \\
\hline Leucoplasia & 9 & 1 & 7 & 0 \\
\hline Língua Geográfica & 2 & 2 & 4 & 0 \\
\hline Líquen Plano & 4 & 3 & 5 & 0 \\
\hline Granuloma Piogênico & 2 & 4 & 8 & 0 \\
\hline Mucocele & 0 & 3 & 6 & 0 \\
\hline
\end{tabular}

Quadro 4: Fatores de risco para o câncer oral e o grau de importância sobre a sua etiologia, segundo avaliação dos dentistas entrevistados.

Quanto à conduta diante de um paciente exposto a fatores de risco do câncer bucal, 09 $(81,8 \%)$ entrevistados disseram que orientam o paciente sobre os perigos destes fatores de risco, enquanto 02 profissionais $(18,2 \%)$ relataram que não tomam qualquer atitude, pois, na concepção deles a exposição aos fatores de risco não representa necessariamente que este paciente desenvolverá câncer oral.

Por outro lado, 10 (90,9\%) entrevistados disseram que tem conhecimento sobre os fatores de risco ligados a patologia citada, enquanto $01(9,1 \%)$ dentista relatou ter conhecimento insuficiente para os reais fatores de risco.

A pesquisa mostrou que $100 \%$ dos dentistas, durante a anamnese, questionam se os pacientes fazem ingestão de álcool e cigarro constantemente, pois a Secretaria Municipal de Saúde do Município disponibiliza uma fi- cha padrão de anamnese para todos os dentistas que trabalham na Atenção Primária onde esse questionamento sobre o consumo de tabaco e álcool está incorporado.

A fala a seguir explicita esta situação: “(...) na ficha de anamnese que a Prefeitura nos disponibiliza tem um dos tópicos que fala sobre o consumo de tabaco, pergunta se o paciente fuma, (...)". Nesse sentido, Horowitz et al. (2000) e Kujan et al. (2006) relataram em estudo que encontraram este percentual para álcool em $60 \%$ e $58,8 \%$ e para fumo em $90 \%$ e $87,4 \%$, respectivamente.

Com referência à etiologia do câncer bucal e o grau de importância de cada condição oral, no que diz respeito à evolução do câncer bucal, todos os pesquisados (100\%) disseram que a associação entre tabaco e álcool está diretamente relacionada com o aparecimento 
do câncer de boca. As respostas dos dentistas pesquisados estão de acordo com estudo de Neville (1998) que demonstra que o tabaco está fortemente ligado ao surgimento de câncer oral e, quando associado ao álcool, o risco aumenta. Segundo Neville (1998) as lesões precursoras do câncer bucal mais importantes, entre as citadas no questionário, são a leucoplasia e a eritroplasia. Apenas um tipo de líquen plano, o líquen plano erosivo, segundo Neville (1998), tem potencial de transformação em malignidade, $01 \%$ a $04 \%$ de chance de transformação em tumor maligno. Nessa pesquisa a leucoplasia e a eritroplasia foram as mais citadas como intrinsecamente relacionadas como precursoras do carcinoma bucal, citados por $81,81 \%$ e $63,63 \%$ respectivamente.

Podemos constatar que o grau de conhecimento dos dentistas pesquisados sobre os reais fatores de risco para o câncer bucal é regular. A maioria relatou que questiona os pacientes durante a anamnese sobre um dos fatores de risco mais importantes sobre câncer bucal, o tabaco e sua associação com o álcool, porém, a radiação solar, diretamente associada ao surgimento queilite actínia, que junto ao tabaco é o principal fator de risco, não é citado por nenhum dentista nas perguntas abertas.

Não existem estudos que relacionem o acometimento de aftas ao câncer bucal, embora apenas $01(9,09 \%)$ dentista respondeu ser sem importância a relação entre ambas, os demais classificaram a relação entre ambas importante ou moderadamente importante; 06 dentistas (54,54\%) entendem como importante ou moderadamente importante a relação entre o aparecimento de granuloma piogênico, embora, também, não existam estudos científicos que mostrem laço entre ambas as patologias. O mesmo se aplica entre infecções bacterianas e trauma, embora $08(72,72 \%)$ e $10(90,90 \%)$ dentistas considerem importante ou moderadamente importante a relação entre ambas, respectivamente.

\subsubsection{Subcategoria: Tecidos moles}

Arguidos sobre a frequência com a qual realizavam exame nos tecidos moles da cavidade oral dos pacientes nas consultas iniciais, $09(81,8 \%)$ dos entrevistados disseram que sempre inspecionam os tecidos moles da ca- vidade oral, $01(9,1 \%)$ relatou que inspeciona ocasionalmente e $1(9,1 \%)$ relatou que inspeciona apenas quando há queixa do paciente. A justificativa entre aqueles que relataram não inspecionar sempre os tecidos moles da cavidade oral foi de que não acham necessário tal procedimento em consultas iniciais, conforme retrata a fala: “(...) caso o paciente sinta alguma alteração ele nos dirá ao longo o tratamento."

Horowitz et al. (2000) em estudo sobre a frequência com que dentistas pesquisados realizavam exame em tecidos moles da cavidade oral nas consultas iniciais constatou-se que $81 \%$ dos pesquisados realizavam este tipo de rastreamento para detecção de algum tipo de alteração. Este estudo coincide com o trabalho apresentado, pois 90\% dos dentistas que trabalham no Programa de Saúde da Família em Resende relataram que rotineiramente realizam este tipo de inspeção.

Estudo de Kujan et al. (2006) constatou que mais de $81,81 \%$ de dentistas pesquisados realizavam exame nos tecidos moles dos pacientes rotineiramente. Este trabalho traz percentagem similar aos trabalhos de Horowitz et al. (2000) e Kujan et al. (2006) sobre a rotina de exames nos tecidos moles dos pacientes realizados pelos dentistas. Apenas um dentista relatou que só realiza este tipo de exame quando solicitado pelo paciente e que não faz parte de sua rotina inspecionar os tecidos moles.

\subsubsection{Subcategoria: Competência dos CDs das ESB em relação ao câncer bucal}

Os 11 dentistas entrevistados (100\%) disseram que é função das Equipes de Saúde Bucal dos Módulos de Saúde da Família o incentivo e a produção de práticas ligadas a eliminação ou diminuição dos fatores de risco, conforme fala selecionada; “(...) aqui nós estamos mais próximos do paciente, da família, criamos vínculos com a comunidade e os agentes comunitários de saúde podem avisar na casa do pacinete para ele vir ao dentista."

Dos CDs entrevistados 100\% relataram não ser papel da atenção primária o diagnóstico final de lesões suspeitas de câncer oral e sim o cadastramento das famílias adscritas em seu território, rastreamento de pacientes com fatores de risco, diagnóstico precoce de lesões sus- 
peitas, encaminhamento para outros níveis de complexidade do sistema de saúde municipal e acompanhamento do paciente e das famílias.

\subsection{Tema: Estrutura dos serviços}

\subsubsection{Categoria: fluxo do paciente de Saúde Bucal}

Como citado anteriormente, Resende possui um CEO, que funciona como centro de referência para as unidades primárias distribuidas no Município. Os pacientes atendidos pelo CEO têm como porta de entrada exclusiva as unidades primárias de saúde e, excepcionalmente, encaminhamentos oriundos do Hospital de Emergência.

Esta frase é bem significativa e esclarecedora: "O CEO atende os pacientes que chegam com a guia de referência da unidade básica de saúde ou, menos comum, do plantão do Hospital de Emergência, mas o paciente tem que ter a guia de referência."

\subsubsection{Subcategoria: encaminhamento}

Os pacientes são encaminhados ao CEO por meio de uma ficha padronizada de encaminhamento para as especialidades. Os pacientes encaminhados com lesões suspeitas de malignidade são referenciados para os dentistas especialistas em cirurgia buco maxilo-facial no CEO. Nestes pacientes com suspeita de câncer bucal é realizada biópsia incisional ou excisional no Centro de Referência e o material é encaminhado para um laboratório conveniado com a Secretaria Municipal de Saúde.

Quanto aos exames complementares, são realizados no único laboratório próprio da Prefeitura. Com o diagnóstico de câncer bucal e de posse da biópsia, exame de lâmina e bloco de cera, este paciente é encaminhado ao Instituto Nacional do Câncer, na Cidade do Rio de Janeiro. A Prefeitura disponibiliza ambulância para o transporte do paciente. A falta de um especialista em Estomatologista no CEO foi citada por um dos pesquisados como um limitador da rede de cuidado em saúde do Município: "não há especialista em Estomatologista, quem faz o procedimento é o buco maxilo".
Foi relatado pelo dentista entrevistado no Centro de Referência que "se o resultado da biópsia der positivo para câncer de boca, o CEO entra em contato com o paciente por telefone e, caso não obtenha resposta, o CEO entra em contato com o PSF mais próximo de sua casa e o agente comunitário de saúde avisa o paciente para ir ao $\mathrm{CEO}$ o mais rápido possível buscar o resultado e conversar com o dentista. O PSF é fundamental nestes casos."

Durante a entrevista, os 2 dentistas especialistas em cirurgia relataram que os pacientes são atendidos somente com a ficha de referência que recebem das unidades primárias de saúde, inclusive dos PSFs, e após atendimento no CEO eles são contra referenciados para as unidades de saúde que os acompanham.

\subsection{Tema: dificuldades para diagnóstico e tratamento das lesões bucais}

\subsubsection{Subcategoria: resolutividade e continuidade do cuidado nas ESB/PSF}

Ao serem questionados como poderia ser melhorado o atendimento, a continuidade do cuidado e o aumento da resolubilidade dos casos, evitando o encaminhamento para especialistas- 07 dentistas $(63,63 \%)$ relataram que as unidades poderiam dar maior resolubilidade e segundo relato "(...) a expansão de exames de auxílio nas unidades, como por exemplo, um aparelho de RX para que a gente possa encaminhar menos para o CEO."

Para todos os dentistas pesquisados (100\%), a questão da territorialidade é um dos pilares da operacionalidade do trabalho das equipes das Unidades de Saúde da Família e do vínculo profissional-paciente estabelecidos, o que permite uma política estratégica para a detecção precoce desses agravos, porém, segundo os entrevistados, é de suma importância que as unidades de Saúde da Família ofertem aos usuários, por estes profissionais, majoritariamente generalistas, a maior variedade de serviços de saúde possível pela atenção básica

A pouca resolubilidade das unidades primárias e o constante encaminhamento para centros de referência conduz o paciente a uma lógica hegemônica fortemente baseada no hospital-especialista-doença. "Alguns pacien- 
tes vêm aqui só para pegar a guia de referência achando que no CEO eles podem realizar todo o tratamento dentário, ainda é muito forte na população a ideia do especialista.”

Outro desdobramento da baixa resolubilidade no PSF é a possibilidade de demora no diagnóstico de lesões suspeitas de malignidade por causa do mecanismo de marcação, embora os dentistas tenham relatado que o fluxo de referência e contra- referência das unidades de PSF para o centro de especialidade exista de maneira estruturada. $\mathrm{O}$ inconveniente desse modelo de sistema de referência e contra referência existente no município, cujos serviços de saúde não estão informatizados e articulados em rede, diz respeito à transferência da responsabilidade pela marcação das consultas para os usuários, gerando transtornos e gastos adicionais com transporte, podendo levar a demora do diagnóstico precoce.

Dar meios e ferramentas para que os dentistas generalistas possam atender esse usuário do sistema de saúde com maior resolubilidade possível fortalece a importância com que e o usuário enxerga a atenção primária, dá agilidade no diagnóstico precoce e minimiza o sofrimento em caso de confirmação do diagnóstico. Aumentar a resolubilidade da atenção primária salva vidas.

\section{Conclusão}

A primeira observação diz respeito à cobertura da população de Resende pelo ESB, que é de 45,94\%. Através da análise dos dados, constata-se que é importante uma ampliação da rede de saúde no que se refere a cobertura de Equipes de Saúde Bucal, para que o rastreamento de lesões suspeitas de câncer oral possa se dar com maior precocidade possível, inclusive por determinação da Portaria Interministerial $n^{\circ}$ 648, de 28 de Março de 2006, que estabelece como Especificidades do Programa de Saúde da Família a "reorganização da Atenção Básica no País, de acordo com os preceitos do Sistema Único de Saúde." Além dos princípios gerais da Atenção Básica, as Equipes de Saúde Bucal devem “(...) ter caráter substitutivo em relação à rede de Atenção Básica tradicional nos territórios em que as Equipes Saúde da Família atuam." (BRASIL, 2006).

Em relação à dedicação dos profissionais ao PSF, o trabalho identificou que a maioria dos dentistas das Equipes de Saúde Bucal pesquisados, o que corresponde a 06 dentistas $(54,55 \%)$, trabalham exclusivamente no Módulo de Saúde da Família, não possuindo qualquer outro tipo de atividade de trabalho.

Destes profissionais que se dedicam exclusivamente ao Programa de Saúde da Família (06 dentistas), a maioria, isto é, 05 dentistas $(83,33 \%)$, é concursada e classificada como estatutária, tendo sido aprovada em concurso específico para o PSF. Esse dado é importante pois o Pacto pela Saúde ${ }^{3}$ tem como um de seus objetivos para o fortalecimento da Atenção Primária “(...) aprimorar a inserção dos profissionais da Atenção Básica nas redes locais de saúde, por meio de vínculos de trabalho que favoreçam o provimento e fixação dos profissionais."

Quanto à capacitação institucional em relação ao câncer bucal, a pesquisa constatou que os dentistas das Equipes de Saúde Bucal têm capacitação institucional periódica promovida pela Prefeitura Municipal de Resende, embora não tenha ocorrido um capacitação específica sobre câncer de boca. Essa informação é importante, tendo em vista que as equipes de SB têm como atribuição fazer o rastreamento dessa patologia. Pode-se concluir que o Município cumpre o preconizado pelo Pacto pela Saúde, que estabelece entre os gestores do SUS:

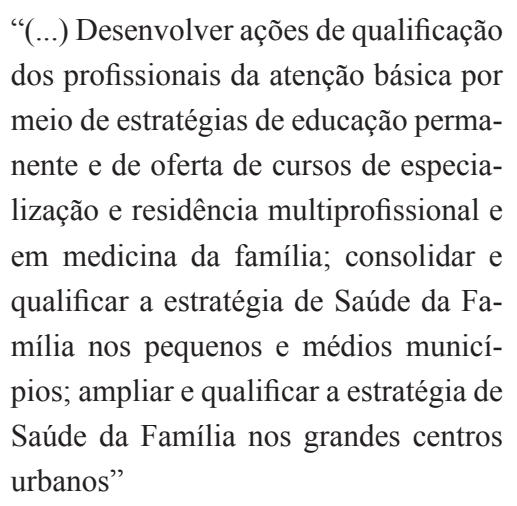

e tem como diretriz: 
"avançar na implementação da Política Nacional de Educação Permanente por meio da compreensão dos conceitos de formação e educação permanente para adequá-los às distintas lógicas e especificidades; considerar a educação permanente parte essencial de uma política de formação e desenvolvimento dos trabalhadores para a qualificação do SUS e que comporta a adoção de diferentes metodologias e técnicas de ensinoaprendizagem inovadoras, entre outras coisas; considerar a Política Nacional de Educação Permanente na Saúde uma estratégia do SUS para a formação e o desenvolvimento de trabalhadores para o setor, tendo como orientação os princípios da educação permanente"

e sendo de responsabilidade do Município:

"formular e promover a gestão da Educação Permanente na Saúde e processos relativos à mesma, orientados pela integralidade da atenção à saúde, criando quando for o caso, estruturas de coordenação e de execução da política de formação e desenvolvimento, participando no seu financiamento."

Em relação às condições de logística, o presente trabalho constatou que a maioria dos dentistas das Equipes de Saúde Bucal pesquisados, o que corresponde a 10 dentistas $(90,90 \%)$, dos Módulos de ESB, relataram que as unidades lhes oferecem boas condições físicas, de material e de insumos para um bom atendimento clínico e de uma boa investigação para rastreamento de lesões pré-malignas ou malignas. Esse dado é importante, pois demonstra que a Secretaria Municipal de Saúde está em sintonia com a Portaria $n^{\circ}$ 648, de 28 de Março de 2006, que estabelece que cabe ao gestor municipal do SUS, "garantir infraestrutura necessária ao funcionamento das Unidades Básicas de Saúde, dotando-as de recursos materiais, equipamentos e insumos suficientes para o conjunto de ações propostas." (Brasil, 2006)

Este trabalho constatou que todos (100\%) os dentistas das Equipes de Saúde Bucal pesquisados ao suspeitarem de lesão pré-cancerígena simplesmente encaminham para o Centro de Especialidade Odontológica, o que está de acordo com a Portaria Ministerial 648 que diz que é papel do dentista do PSF “encaminhar e orientar os usuários a outros níveis de complexidade do sistema e principalmente, manter a responsabilização pelo acompanhamento do usuário e o seguimento do tratamento, dentre outras responsabilidades" (Brasil, 2006), entretanto, a baixa resolutividade das Equipes de Saúde da Bucal pesquisadas compromete a realização da

“(...) atenção integral em saúde bucal, como a promoção e proteção da saúde, prevenção de agravos, diagnóstico, tratamento, reabilitação e manutenção da saúde individual e coletiva a todas as famílias, a indivíduos e a grupos específicos, de acordo com planejamento local, com resolubilidade." (BRASIL, 2006)

Pode-se ressaltar quanto à marcação de consultas que tanto os dentistas das Equipes de Saúde Bucal quanto os dentistas do CEO, responsáveis pelo diagnóstico, consideram o sistema de referência e contra referência do Município de Resende como operacional e de bom funcionamento, porém, o constante deslocamento dos usuários para os centros de referências e a impossibilidade da realização de uma série de exames complementares para fins de diagnóstico em unidades próximas a sua residência, além de transtornos e gastos adicionais com transporte, submete o usuário a uma lógica de cuidado em saúde em que somente o especialista localizado no hospital ou no centro de referência pode dar resolubilidade ao seu agravo, comprometendo inclusive o diagnóstico precoce. O aperfeiçoamento do sistema de referência e contra referência é fundamental para a concretização do conceito da integralidade.

Como os Centros de Especialidades Odontológicas são preparados para ofertar à população o diagnóstico bucal, com ênfase no diagnóstico e detecção do câncer oral, verificou-se, diante do exposto, que a falta de um profissional especialista em Estomatologia no Centro de Referência do Município compromete um dos pilares do próprio Centro de Referência.

Em suma, para aperfeiçoamento nos processos de ampliação da rede de saúde e da continuidade do cuidado é importante as Equipes 
de Saúde da Família/Equipes de Saúde Bucal ampliarem a oferta de serviços de saúde aos usuários do sistema cadastrados no território do PSF. A ampliação de serviços de saúde pela Atenção Primária, e, consequentemente, o aumento da resolutividade, evitando transtornos adicionais aos pacientes e dando mais agilidade ao diagnóstico, pode, efetivamente, contribuir para a qualidade na sobrevida dos pacientes com diagnóstico positivo de câncer oral.

\section{Referências}

ALONGE O. K., NARENDRAN S., Opinions about oral câncer prevention and early detection among dentists practicing along the Texas-Mexico border. Oral Dis, v. 9, n. 1, p. 41-45, 2003.

ANTUNES, J. L. F.; TOPORCOV, T. N.; WUNSCH-FILHO, V.. Resolutividade da campanha de prevenção e diagnóstico precoce do câncer bucal em São Paulo, Brasil. Revista Panamericana de Salud Pública, Brasília, v. 21, n. 1, p. 52-60, jan. 2007.

BRASIL. Ministério da Saúde. Departamento de Atenção Básica. Saúde Bucal. Brasília: Ministério da Saúde, 2008. (Cadernos de Atenção Básica, n. 17)

Instituto Nacional de Câncer.

Estimativa 2005: incidência de câncer no Brasil. Rio de Janeiro: INCA, 2004.

Ministério da Saúde. Lei $n^{\circ} 8080$, de 19 de setembro de 1990. Diário Oficial da União, Poder Executivo, Brasília, 20 set. 1990. Seção 1. Disponível em: http://www. saude.inf.br/legisl//lei8080.htm>. Acesso em: 11 nov. 2009.

.Ministério da Saúde. Instituto Nacional do Câncer. Estimativas de Incidência de Câncer no Brasil para 2006: câncer de boca. Rio de Janeiro: Mistério da Saúde, 2007.

SIAB: manual do sistema de informação da atenção básica. Brasília, MS, 1998.
Secretaria Nacional de Programas Especiais de Saúde. Divisão Nacional de Saúde Bucal. Política Nacional de Saúde Bucal: Princípios, Objetivos, Prioridades. Brasília: Ministério da Saúde, 2004.

Ministério da Saúde. Secretaria de Assistência à Saúde. Instituto Nacional do Câncer. Falando sobre câncer de boca. Rio de Janeiro: INCA; 2002.

Ministério da Saúde. Portaria/ MS 648 de 28 de Março de 2006. Aprova a Política Nacional de Atenção Básica, estabelecendo a revisão de diretrizes e normas para a organização da Atenção Básica para o Programa Saúde da Família (PSF) e o Programa Agentes Comunitários de Saúde (PACS). Diário Oficial da Republica Federativa do Brasil, Brasília, 29 mar. de 2006.

BUNDGAARD, T.; BENTZEN, S. M.; WILDT, J. The prognostic effect of tobacco and alcohol consumption in intra-oral squamous cell carcinoma. Eur J Cancer B Oral Oncol, Wasghington, v. 30B, n. 5, p. 323-8, 1994.

CARVAlHO, M. B. et al. Características clínico-epidemiológicas do carcinoma epidermóide de cavidade oral no sexo feminino. Rev Assoc Med Bras, São Paulo, v. 47, n. 3, p. 208-14, 2001.

CERVI A, HERMSDORFF HHM, RIBEIRO RCC. Tendência da mortalidade por doenças neoplásicas em 10 capitais barsileiras, de 1980 a 2000. Rev Bras Epidemiol, São Paulo, v. 8, n. 4, p. 4007-4018, 2005

CLOVIS J.B., HOROWITZ A.M., POEL D.H. Oral and pharyngeal cancer: practices and opinions of dentists in British Columbia and Nova Scotia. J Can Dent Assoc 2002b;68 (7): 420-5.

COTRAN, R. S.; KUMAR, V.; COLLINS, T. Robbins: Patologia Estrutural e Funcional. Rio de Janeiro: Guanabara Koogan, 2000. p. 151.

DEBS Y.D., SANCHEZ F.B., CARVALHO C.C.D., ARAÚJO S.S. Aspectos atuais da prevenção primária do câncer de mama. Rev Bras Mastol, local, v.10, n.3. p. 22-7, 2000. 
DEDIVITIS, R. A. et al. Características clínicoepidemiológicas no carcinoma espinocelular de boca e orofaringe. Rev Bras Otorrinolaringol, São Paulo, v. 70, n. 1, p. 35-40, 2004.

FERNANDES, S. M., PERES, M. A. Associação entre Atenção Básica em Saúde Bucal e Indicadores Sócio-Econômicos Municipais. Cad. Saúde Pública, Brasília, vol. 39, n. 6, p.(XI), 2005.

GENOVESE, W. J. Câncer de Boca: noções básicas de prevenção e diagnóstico. São Paulo: Ed. Fundação Peirópolis., 2001. p. 1997.

GROISMAN, S.; MORAES, N. M.; CHAGAS, L. D. A evolução da atenção à saúde no Brasil: o contexto da saúde bucal. Cadernos da ABOPREV II, Rio de Janeiro, 2005.

HA, P. K., CALIFANO, J. A. The role of human papillomavirus in oral carcinogenesis.

Crit Rev Oral Biol Med, London, v. 15, n. 4, p. 188-96, 2004.

KOWALSKI L.P., NISHIMOTO I.N. Epidemiologia do Câncer de Boca. In: Parise Jr. O. Câncer de boca: aspectos básicos e terapêuticos. São Paulo: Sarvier; 2000. Cap 1, p. 3-11.

\section{KUJAN O., DUXBURY A.J., GLENNY} A.M., THAKKER N.S., SLOAN P. Opinions and attitudes of the UK's GDP's and specialists in oral surgery, oral medicine and surgical dentists on oral cancer screening. Oral Dis 2006; 12:190-9.

LEÃO J.C., GOÉS P., SOBRINHO C.B., PORTER S. Knowledge and clinical espertise regarding oral câncer among Brasilian dentists. Int J Oral Maxilofac Surg 2005; 34:436-9.

LETÁCIO, C. Do Programa Saúde da Família ao Brasil Sorridente: O caminho da integralidade em saúde bucal. 129 f. Dissertação (Mestrado em Saúde da Família) - Faculdade de Medicina da Universidade Estácio de Sá, Rio de Janeiro, 2009.

MCCANN M. F., MACPHERSON L. M. D., BINNIE V. I., STEPHEN K. W. A survey of Scottish primary care dental practitioners oral cancer-related practicesand trainingrequirements.

Com Dent Health 2000; 17: 24-30.
MARINHO, M. B. O Demônio nos "Paraísos Artificiais": considerações sobre as políticas de comunicação para a saúde relacionada ao consumo de drogas. Interface - Comunicação, Saúde, Educação. Botucatu, v.9, n.17, p.222, Mar./Aug. 2005.

MASHBERG A. Diagnosis of early oral and oropharyngeal aquamous carcinoma: obstacles and their amelioration. Oral Oncol 2000; 36:253-5.

MINAYO, M.C.S. Organizadora. Pesquisa Social: Teoria, Método e Criatividade. $15^{\mathrm{a}} \mathrm{Ed}$. Rio de Janeiro: Vozes, 2000, p. 181.

MERCADANTE, O. A. Evolução das políticas e do sistema de saúde no Brasil. In: FINKELMAN, J. Caminhos da saúde pública no Brasil. Rio de Janeiro: Editora Fiocruz, 2002. Cap 3, p. 237-312.

NEVILLE, B. W., BOUQUOT, J. E., DAMM, D. D., ALLEN, C. M. Patologia Oral e Maxilofacial. $1^{\circ}$ ed. Rio de Janeiro: Editora Guanabara Koogan, 1998.

NUNES M.O., TRAD L.B., ALMEIDA B.A., HOMEM C.R., MELO M.C.I.C. O Agente Comunitário de Saúde: construção da identidade desse personagem híbrido e polifônico. Cad Saúde Pública, Brasília, v. 18, n. 6, p. 1629-1638, 2002.

OLIVEIRA C.E., BERNINI G.F., MIYAZAKI L.C.Y., TOMITA N.E. Características sociodemográficas da mortalidade por câncer de boca em Bauru, SP, no período de 1991 a 2001: uso de geoprocessamento. Rev. bras. epidemiol. São Paulo, v.11, n.2, p. 15-23, 2008.

ONIZAWA K., NISHIHARA K., YAMAGATA K., YUSA H., YANAGAWA T., YOSHIDA H. Factors associated with diagnostic delay of oral squamous cell carcinoma. Oral Oncol 2003; 39:781-8.

ORTEGA-ALTAMIRANO, D.; LÓPEZCARRILLO, L.; LÓPEZ-CERVANTES, M. Strategies for breast selfexamination training to women of reprodutctive age. Salud Pública Mex, México, v. 42, n. 1, p. 17-25, 2000. 
PINHEIRO, G. C. D. et al. Prevenção do câncer bucal. Bragança Paulista: Encadernadora Pietro, 1998.

PINTO, V. G. Características do subsistema público federal de prestação dos serviços em Odontologia. Brasília: Tese de mestrado apresentada à Faculdade de Saúde Pública da USP, 1977. 171f.. Dissertação (Mestrado em Saúde Coletiva) - Instituto Saúde Pública da Universidade de São Paulo, São Paulo, 1977.

RONCALLI, A. G. et al. Modelos assistenciais em saúde bucal no Brasil: tendências e perspectivas. Ação Coletiva, v.2, p. 9-14, 1999.

SERRA, C. G. A Saúde Bucal como Política de Saúde. Análise de três experiências recentes: Niterói, Campinas e Curitiba. 1998. 132 f. Dissertação (Mestrado em Saúde Coletiva) Universidade Estadual do Rio de Janeiro, UERJ, 1998.

SERRA, C. G. Garantia de acesso à atenção básica e continuidade de cuidados como estratégias para consolidação da integralidade no SUS: uma análise sobre os processos de implantação do PSF, construção de sistemas de referência e regionalização assistencial no Estado do Rio de Janeiro. 2003. 203 f. Tese (Doutorado em Saúde Coletiva) Universidade Estadual do Rio de Janeiro, UERJ, 2003.

SOUZA H.M., SAMPAIO L.F.R. Atenção básica: políticas, diretrizes e modelos coetâneos no Brasil. In: Negri B, Faria R, Viana ALD, organizadores. Recursos Humanos em Saúde: política, desenvolvimento e mercado de trabalho. Campinas: Instituto de Economia/ UNICAMP, 2002. p.9-32.
TRALONGO, V. et al. Prognostic factors in oral squamous cell carcinoma. A review of the literature. Anticancer Res, New York, v. 19, n. 4C, p. 3503-10, 1999.

TOBAR, F., YALOUR, M. R. Como fazer teses em saúde pública: conselhos e idéias para formular projetos e redigir teses e informes de pesquisa. Rio de Janeiro: Fiocruz, 2001, p.111.

VASCONCELOS, E. M. Comportamento dos cirurgiões-dentistas das unidades básicas de saúde do município de São Paulo quanto a prevenção e ao diagnóstico precoce do câncer bucal. 102 f. Dissertação (Mestrado em Odontologia) - Universidade de São Paulo, São Paulo, 2006.

\section{VIANA, M.I.P. Estado e atenção odontológica} no Brasil: um estudo sobre as políticas de saúde bucal na conjuntura pós-74. 153 f. Dissertação (Mestrado em Ciências da Saúde)- Faculdade de Odontologia da Universidade Federal da Bahia, Salvador, 1988.

ZANETTI, C. H.. As marcas do mal-estar social no Sistema Nacional de Saúde: o caso das políticas de saúde bucal, no Brasil dos anos 80. 110 f. Dissertação (Mestrado em Ciências da Saúde) - Escola Nacional de Saúde Pública, Rio de Janeiro, 1993.

ZANETTI, C. H.. A utilidade como função para universalidade e eqüidade: uma análise formal da validade instrumental do ordenamento administrativo federal da assistência à saúde bucal no saúde da família. 210 f. Tese (Doutorado em ciências da Saúde) - Escola Nacional de Saúde Pública, Rio de Janeiro, 2005.

\author{
Endereço para Correspondência: \\ Sylvio da Costa Júnior \\ sylviocosta13@hotmail.com \\ Quadra 2 - Conjunto A-05 - Bloco B - Apto. 303 \\ Sobradinho - Brasília - DF \\ CEP: $73.015-105$
}

Informações bibliográficas:

Conforme a NBR 6023:2002 da Associaçao Brasileira de Normas Técnicas (ABNT), este texto científico publicado em periódico eletrônico deve ser citado da seguinte forma: JÚNIOR, SYLVIO DA COSTA; SERRA CARLOS GONÇALVES. Diagnóstico e Continuidade do Cuidado do Câncer Bucal em pacientes acompanhados pelas Equipes de Saúde Bucal do Programa de Saúde da Família: a experiência do município de Resende, no Estado do Rio de JaneiroCadernos UniFOA. Volta Redonda, Ano VI, n. 15, abril 2011. Disponivel em: <http://www.unifoa.edu.br/cadernos/edicao/15/73.pdf> 